\title{
Fstl1 Promotes Glioma Growth Through the BMP4/Smad1/5/8 Signaling Pathway
}

\author{
Xin Jin $^{\mathrm{a}} \quad$ Er Nie $^{\mathrm{a}} \quad$ Xu Zhou ${ }^{\mathrm{a}} \quad$ Ailiang Zeng $^{\mathrm{a}} \quad$ Tianfu Yu $^{\mathrm{a}} \quad$ Tongle Zhi $^{\mathrm{a}} \quad$ Kuan Jiang $^{\mathrm{a}}$ \\ Yingyi Wang $^{\mathrm{a}}$ Junxia Zhang ${ }^{\mathrm{a}}$ Yongping You ${ }^{\mathrm{a}, \mathrm{b}}$ \\ aDepartment of Neurosurgery, the First Affiliated Hospital of Nanjing Medical University, Nanjing, \\ China, ${ }^{b}$ Chinese Glioma Cooperative Group (CGCG), China
}

\section{Key Words}

Glioblastoma $•$ Proliferation $•$ Fstl1 $・$ BMP4 $・$ Smad1/5/8 signaling pathway

\begin{abstract}
Background: Gliomas result in the highest morbidity and mortality rates of intracranial primary central nervous system tumors because of their aggressive growth characteristics and high postoperative recurrence. They are characterized by genetic instability, intratumoral histopathological variability and unpredictable clinical behavior in patients. Proliferation is a key aspect of the clinical progression of malignant gliomas, complicating complete surgical resection and enabling tumor regrowth and further proliferation of the surviving tumor cells. Methods: The expression of Fstl1 was detected by western blotting and qRT-PCR. We used cell proliferation and colony formation assays to measure proliferation. Then, flow cytometry was used to analyze cell cycle progression. The expression of Fstl1, p-Smad1/5/8 and p21 in GBM tissue sections was evaluated using immunohistochemical staining. Furthermore, we used coimmunoprecipitation (Co-IP) and immunoprecipitation to validate the relationship between Fstl1, BMP4 and BMPR2. Finally, we used orthotopic xenograft studies to measure the growth of tumors in vivo. Results: We found that follistatin-like 1 (Fstl1) was upregulated in highgrade glioma specimens and that its levels correlated with poor prognosis. Fstl1 upregulation increased cell proliferation, colony formation and cell cycle progression, while its knockdown inhibited these processes. Moreover, Fstl1 interacted with bone morphogenetic protein (BMP) 4, but not BMP receptor (BMPR) 2, and competitively inhibited their association. Furthermore, Fstl1 overexpression suppressed the activation of the BMP4/Smad1/5/8 signaling pathway, while BMP4 overexpression reversed this effect. Conclusion: Our study demonstrated that Fstl1 promoted glioma growth through the BMP4/Smad1/5/8 signaling pathway, and these findings suggest potential new glioblastoma treatment strategies.
\end{abstract}

$X$. Jin and E. Nie contributed equally to this work.

Yongping You

KARGER
Department of Neurosurgery, The First Affiliated Hospital of Nanjing Medical University Nanjing, Jiangsu Province, (China)

Tel. +86 025 83718836, Fax +86025 83718836, E-Mail yypl9@njmu.edu.cn 


\section{Cellular Physiology Cell Physiol Biochem 2017;44:1616-1628

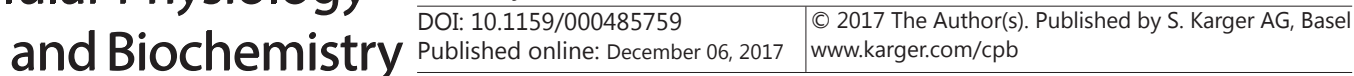 \\ Jin et al.: Fst11-BMP4-Smad1/5/8 Signaling}

\section{Introduction}

Glioblastoma multiforme (GBM) is the most common and deadly brain tumor in adults, accounting for approximately $80 \%$ of malignant tumors affecting the central nervous system $[1,2]$. Despite advances in standard therapy, including diagnostic methods and treatment strategies, the prognosis for patients with GBM remains poor, with an average survival time of 12-15 month after diagnosis [3-5]. Therefore, new anticancer agents for treating this disease are urgently needed.

Bone morphogenetic proteins (BMPs) are multifunctional growth factors belonging to the transforming growth factor beta (TGF- $\beta$ ) superfamily [6]. Transforming growth factor $\beta$ (TGF $\beta$ ) receptor signaling pathways play essential and diverse roles in the regulation of many biological events, including cell replication, differentiation, apoptosis and tissue remodeling [7]. The well-established functional role of BMPs in development and their ability to regulate vital cellular processes have made them an increasingly interesting topic in cancer research, and a wealth of new information has been reported regarding the role of BMPs in cancer pathogenesis. BMPs are aberrantly expressed in multiple malignancies. Manipulations of BMP signaling have been shown to cause distinct changes in the phenotype of cancer cells [810]. BMPs have been grouped into 15 categories and further divided into three subsets based on their amino acid sequences. The three subsets are BMP2 and BMP4, BMP5 and BMP8 and BMP3 and GDF10[11]. In recent years, some members of the BMP family, especially BMP4, have been found to play a role in cancer pathogenesis [12]. According to these studies, BMP4 expression levels in different tumors usually vary. Compared to levels in corresponding normal tissues, BMP4 expression was increased in gastric, hepatocellular, and colorectal carcinomas [13-16]. In contrast, reduced BMP4 expression was observed in pituitary tumors and meningiomas [14,17]. In most tumor types, BMP4 inhibits cancer cell growth, but opposing or even conflicting results have also been reported. For example, reduced cell growth after BMP4 treatment or overexpression was observed in cell lines derived from basal cell carcinomas and myeloma, breast, gastric, lung, and pancreatic cancers $[18,19]$. It has been reported that in myeloma and retinoblastoma, BMP4 could reduce cell growth and induce small mothers against decapentaplegic 1/5/8 (Smad1/5/8) phosphorylation; these effects were attenuated by cotreatment with 2 BMP type I receptor inhibitors (dorsomorphin and DMH-1)[20, 21]. Recent studies found that BMP4 can inhibit the growth of glioma cells. The expression of BMP4 and its receptor (BMPR), both their transcripts and proteins, was detected in GBM cells, and the expression levels were closely related to the WHO grade [22]. To sum up, BMP4 plays an important role in the development of glioma.

Follistatin-like 1 (Fstl1), first identified as a TGF- $\beta 1$ inducible gene, encodes a secreted extracellular glycoprotein that contains a follistatin-like domain in its amino acid sequence [23-25]. According to in silico analysis, Fstl1 has a domain similar to follistatin, suggesting that it plays a role in TGF- $\beta$ superfamily-induced inhibition [26-28]. Database analysis showed that Fstl1 expression was significantly increased in high grade gliomas compared with that in lower grade gliomas and was associated with glioma prognosis [29, 30]. However, the role of Fstl1 in GBM biology remains unknown. In this study, we showed that Fstl1 inhibited Smad1/5/8 phosphorylation by blocking the association of BMP4 and BMPR2 through competitive binding to BMP4. Thus, Fstl1 promoted glioma growth and may be a potential therapeutic target for treating GBMs.

\section{Materials and Methods}

Affymetrix microarray analysis of cell lines and patient tissue specimens

Two expression profiles (GSE4290 and GSE55918) were acquired from the Gene Expression Omnibus (GEO, http://www.ncbi.nlm.nih.gov/geo/) database. From the GSE4290 data, 23 brain tissue samples from epilepsy patients were used as nontumor control samples, and 157 glioma samples, including astrocytoma, oligodendroglioma and glioblastoma (45 grade II, 31 grade III and 81 grade IV), were used. From the 


\section{Cellular Physiology Cell Physiol Biochem 2017;44:1616-1628

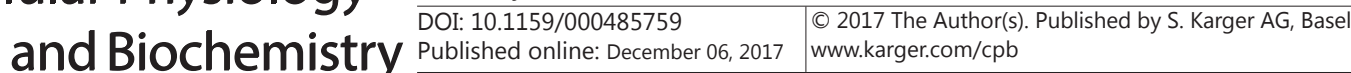

Jin et al.: Fstl1-BMP4-Smad1/5/8 Signaling

GSE55918 data, 15 brain tissue samples from epilepsy patients were used as nontumor control samples, and 1679 glioma samples, including astrocytoma, oligodendroglioma and glioblastoma (11 grade I, 166 grade II, 233 grade III and 1269 grade IV), were used. The original CEL files and probe annotation of the platform were used.

\section{Cell lines and patient tissue specimens}

The human glioblastoma multiforme (GBM) cell lines A172, U87 and LN229 were purchased from the Shanghai Cell Bank of the Chinese Academy of Sciences (Shanghai, China). Normal human astrocytes (NHAs) were obtained from Lonza (Walkersville, MD, USA) and cultured in the provided astrocyte growth media supplemented with rhEGF, insulin, ascorbic acid, GA-1000, L-glutamine and 5\% FBS. The primary GBM2 cell line was established in October 2013 from cells taken from a patient with right tempus glioblastoma. After written patient consent was obtained, tumor tissues were collected from the operating room during resection according to protocols approved by the First Affiliated Hospital of Nanjing Medical University Institutional Review Board. Tissues were obtained from viable tumor regions. Within 2 hours, the samples were either finely minced prior to organoid formation or dissociated into single-cell suspensions. Red blood cells were removed by brief hypotonic lysis; cell numbers were counted, and viability was assessed using trypan blue. Primary cell lines were maintained in primary serum-free cultures grown on laminin. All GBM cell lines were validated in October 2014 by short tandem repeat DNA fingerprinting using the AmpFISTR Identifiler Kit according to the manufacturer's instructions (Applied Biosystems, CA, USA). All GBM cell lines were preserved in liquid nitrogen to maintain authenticity. The cells used for the experiments were replenished from frozen stocks every 3 months. Cells were cultured in $5 \% \mathrm{CO}_{2}$ at $37^{\circ} \mathrm{C}$ in Dulbecco's modified Eagle's medium (DMEM, Gibco, CA, USA) supplemented with 10\% fetal bovine serum (Gibco)

This study was approved by the Institutional Review Board and the Research Ethics Committee of Nanjing Medical University (Nanjing, Jiangsu, China). Informed consent was obtained from all participants. All methods were performed in accordance with the approved guidelines. Plasmid and stable cell line construction and transfection were also conducted in accordance with institutional guidelines.

\section{Plasmid and stable cell line construction and transfection}

The entire coding sequences of Fstl1 and BMP4 were obtained from HUVEC mRNA by RT-PCR. Fstl1 and BMP4 cDNA was purified by Genechem (Shanghai, China) and cloned into the pcDNA3.1-FLAG vector to generate pcDNA3.1-FLAG-Fstl1 and pcDNA3.1-FLAG-BMP4 recombinant plasmids. Transient transfection was performed using the Lipofectamine ${ }^{\mathrm{TM}} 2000$ transfection reagent (Invitrogen) according to the manufacturer's instructions. For stable transduction, GBM cells were transfected with Fstl1, shFstl1, or shBMP4 (Genechem) lentiviral particles according to the manufacturer's protocol. Scrambled lentiviral particles were used as a control. After $48 \mathrm{~h}$ of incubation, the medium was replaced with DMEM containing $5 \mu \mathrm{g} / \mathrm{ml}$ puromycin. After maintenance for 3-4 weeks in selection media, puromycin-resistant colonies were selected and screened for Fstl1 or BMP4 expression.

\section{Quantitative real-time PCR}

Real-time PCR was performed using an iQ5 Multicolor Real-Time PCR Detection System (Bio-Rad, Hercules, CA, USA) according to the manufacturer's instructions. The primers used were as follows: has-Fstl1, forward primer 5' - CCCAGTTGTTTGCTATCAGTC-3',reverse primer 5' - CAGTGTCCATCGTAATCAACCTG-3'; and has-P21, forward primer 5'-TGTCCGTCAGAACCCATGC-3', reverse primer 5'- AAAGTCGAAGTTCCATCGCTC-3'. As an internal control, has-GAPDH mRNA was amplified under the same PCR conditions using forward primer 5'- GGAGCGAGATCCCTCCAAAAT-3' and reverse primer 5'- GGCTGTTGTCATACTTCTCATGG-3'. All experiments were performed in biological triplicate and experimental duplicate. Relative expression values were calculated using the $2-\Delta \Delta \mathrm{Ct}$ method.

\section{Western blotting}

Western blotting was performed as described previously [44], and images were captured with a BioRad ChemiDoc XRS+ system (Bio-Rad, Hercules, CA, USA). The antibodies used were targeted against the following: Fstl1 and BMP4 (Abcam, Cambridge, MA, USA); BMPR2 and p21 (Cell Signaling Technology, USA); and Smad1/5/8 and p-Smad1/5/8 (Santa Cruz, USA). An antibody against GAPDH (Cell Signaling Technology) was also used as a control. 


\section{Cellular Physiology Cell Physiol Biochem 2017;44:1616-1628 \begin{tabular}{ll|l} 
DOI: 10.1159/000485759 & $\begin{array}{l}\text { O 2017 The Author(s). Published by S. Karger AG, Basel } \\
\text { www.karger.com/cpb }\end{array}$
\end{tabular} Jin et al.: Fst11-BMP4-Smad1/5/8 Signaling}

\section{Cell proliferation assay}

Cells in the logarithmic phase of growth were seeded at 3000 cells per well and cultured in 96-well plates. Cell proliferation was assessed based on the assay results at the indicated time points using a Cell Counting Kit-8 (CCK8, Dojindo Laboratories, Japan) according to the manufacturer's instructions.

Flow cytometry for cell cycle analysis

Transfected cells were harvested, washed with PBS and fixed in 70\% ice-cold ethanol. Fixed cells were resuspended in Cell Cycle Staining Kit solution (Multi Sciences, Hangzhou, China), incubated for 30 min in the dark and then analyzed by flow cytometry.

\section{Colony formation assay}

Cells stably expressing Fstl1, sh Fstl1-7 or the vector (control) were independently plated on 60-mm plates ( 200 cells per $60-\mathrm{mm}$ plate). After 10 to $12 \mathrm{~d}$, colonies were fixed in $100 \%$ methanol and stained with $0.1 \%$ crystal violet in $20 \%$ methanol for 15 min. Colony-forming efficiency was calculated as the number of visible colonies/plated cells $\times 100 \%$.

\section{Co-immunoprecipitation (Co-IP)}

Co-immunoprecipitation assays were performed using a co-immunoprecipitation kit (Thermo Scientific, Waltham, MA, USA). GBM cells were lysed in SDS lysis buffer on ice for $30 \mathrm{~min}$, and the supernatants were collected after centrifugation at $4^{\circ} \mathrm{C}$ and $14,000 \times \mathrm{g}$ for $15 \mathrm{~min}$. The protein lysates were then incubated with anti-BMP4, anti-BMPR2 or anti-Fstl1 antibody on a rotator overnight at $4^{\circ} \mathrm{C}$. Next, the mixtures were incubated with immobilized protein $\mathrm{A} / \mathrm{G}$ beads (Thermo Scientific) on a rotator for $2 \mathrm{~h}$ at $4^{\circ} \mathrm{C}$. The beads were collected by centrifugation at $3000 \times \mathrm{g}$ for $2 \mathrm{~min}$ and washed five times with $0.5 \mathrm{ml}$ of IP wash buffer. SDS loading buffer was then added to the beads, and the samples were denatured at $95^{\circ} \mathrm{C}$ for $8-10 \mathrm{~min}$. Finally, the supernatants were collected and either stored at $-80^{\circ} \mathrm{C}$ or immediately analyzed by western blotting.

\section{Orthotopic xenograft studies}

Animal experiments were approved by the Animal Management Rule of the Chinese Ministry of Health (documentation 55, 2001) and were performed in accordance with the approved guidelines and experimental protocols of Nanjing Medical University (Nanjing, China). All experiments involving mice were performed by the Model Animal Research Center of Nanjing University (Nanjing, China). For orthotopic xenograft studies, GBM cells $\left(2.5 \times 10^{5}\right)$ stably expressing Fstl1, shFstl1-7 or shCtrl were injected intracranially into the striatum of nude mice using a stereotactic device (coordinates: $2 \mathrm{~mm}$ anterior and 2 $\mathrm{mm}$ lateral to the dura and $3 \mathrm{~mm}$ deep). Mice were sacrificed when they displayed advanced signs of tumor growth (rough coat, hunching, and weight loss). Tumors were measured weekly by luminescence imaging (IVIS Spectrum, PerkinElmer, USA).

Immunohistochemistry (IHC)

Immunohistochemistry to detect Smad1/5/8 in nude mouse xenograft tumor tissues was performed as described previously [31].

\section{Statistical analysis}

Quantitative data are presented as the means \pm SEM. Statistical significance levels were set at $P<$ $0.05\left({ }^{*}\right)$ and $P<0.01(* *)$. One-way ANOVA and Student's t-tests were used to compare groups. Biostatistical analyses were performed using GraphPad Prism 5 software (GraphPad). All statistical tests were two-sided.

\section{Results}

Fstl1 expression was increased in human glioma tissues

To identify the role of Fstl1 in glioma, we analyzed Fstl1 expression using the GSE4290 and GSE55918 databases; we found that Fstl1 expression was significantly increased in highgrade glioma tissue samples, particularly glioblastoma (Figs. 1A-C). This result was verified 


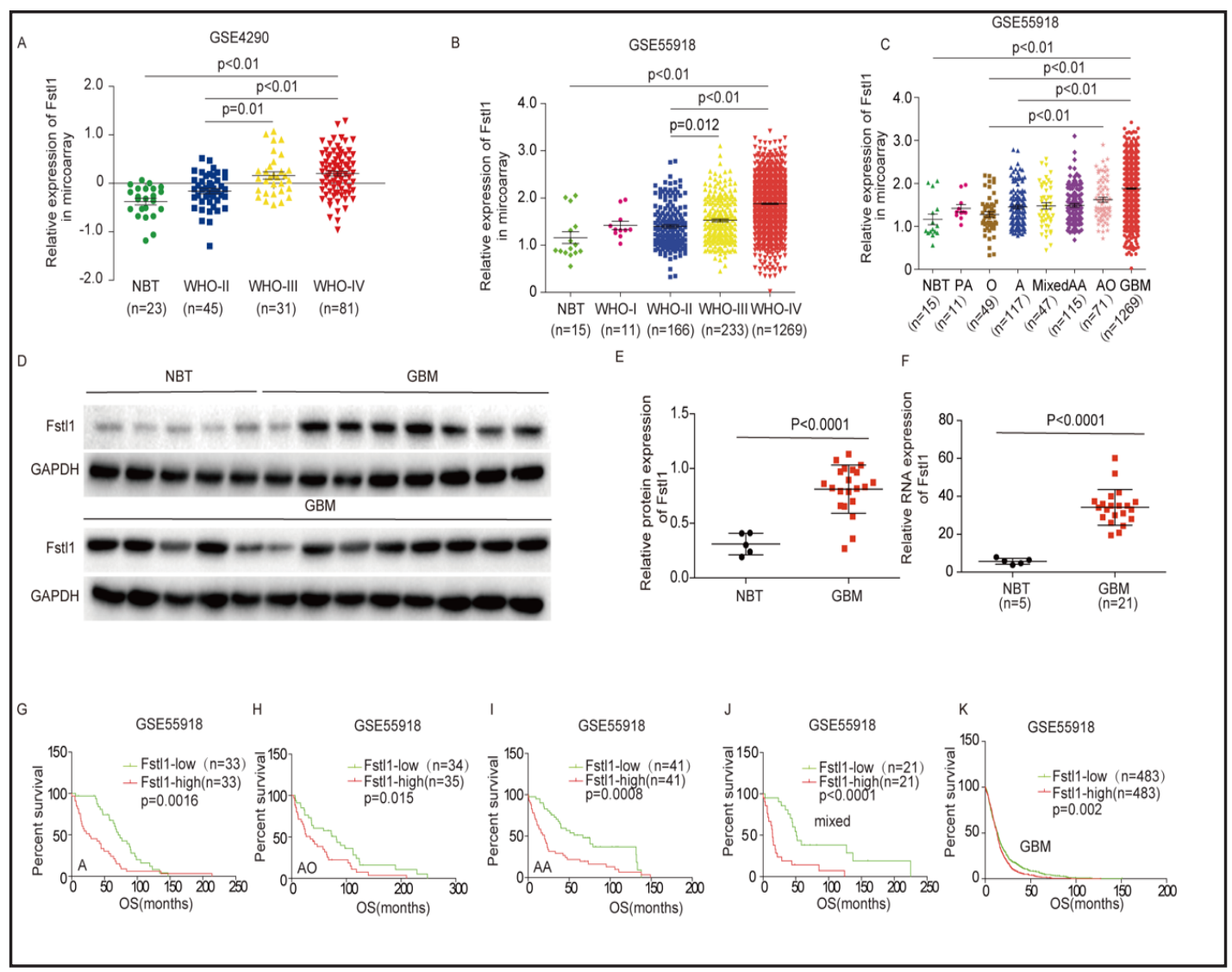

Fig. 1. Fstl1 was upregulated in glioma, and its expression was correlated with low survival rates. (A), Fstl1 levels were analyzed in NBTs and different grades of gliomas from the GSE4290 database. (B) and (C), Levels of Fstl1 were analyzed in NBTs and different grades of gliomas from the GSE55918 database. (D) and (E), Protein levels of Fstl1 in NBTs $(n=5)$ and GBM tissues $(n=21)$ were examined by western blotting. (F) mRNA levels of Fstl1 were assessed by qRT-PCR. (G-K) Kaplan-Meier curves show the overall survival of patients with high or low levels of Fstl1 expression in the glioma tissue samples from the GSE55918 database. A: astrocytoma; AA: anaplastic astrocytoma; AO: anaplastic oligodendroglioma; Mixed: mixed GBM: glioblastoma multiforme; NBT: nontumor brain tissue. Student's t-tests and one-way ANOVA were performed. Data are the means $\pm \operatorname{SEM}\left({ }^{* *} \mathrm{P}<0.01\right.$, \#P>0.05).

by the data obtained from clinical samples (Figs. 1D-E). We also analyzed Fstl1 expression in 21 human glioma tissue samples and five human normal brain tissue samples using qRT-PCR (Fig. 1F). On average, Fstl1 expression was increased in glioma tissue samples, particularly in those from high-grade gliomas. Further analysis of the GSE55918 database showed that high Fstl1 expression levels were correlated with poor survival in patients with gliomas of various grades (Figs. 1G-K).

\section{Fstl1 overexpression promoted cell proliferation and cell cycle progression}

To investigate its role in glioma progression, we analyzed Fstl1 levels in normal human astrocytes (NHAs) and five GBM cell lines (A172, LN229, primary GBM2, U87 and U251 cells) by western blotting. Almost all GBM cells had higher levels of Fstl1 than those in NHAs. Fstl1 expression was the highest in U87 and U251 cells and the lowest in LN229 and primary GBM2 cells (Fig. 2A). We then established LN229 and primary GBM2 cell lines that stably expressed Fstl1 (Fig. 2B). Flow cytometry was used to investigate the role of Fstl1 in glioma progression. Fstl1 overexpression led to an increased number of cells in S phase, while the percentage of cells in G1/G0 phase decreased from 55.7 to $41.9 \%$ in the LN229 cell line and 


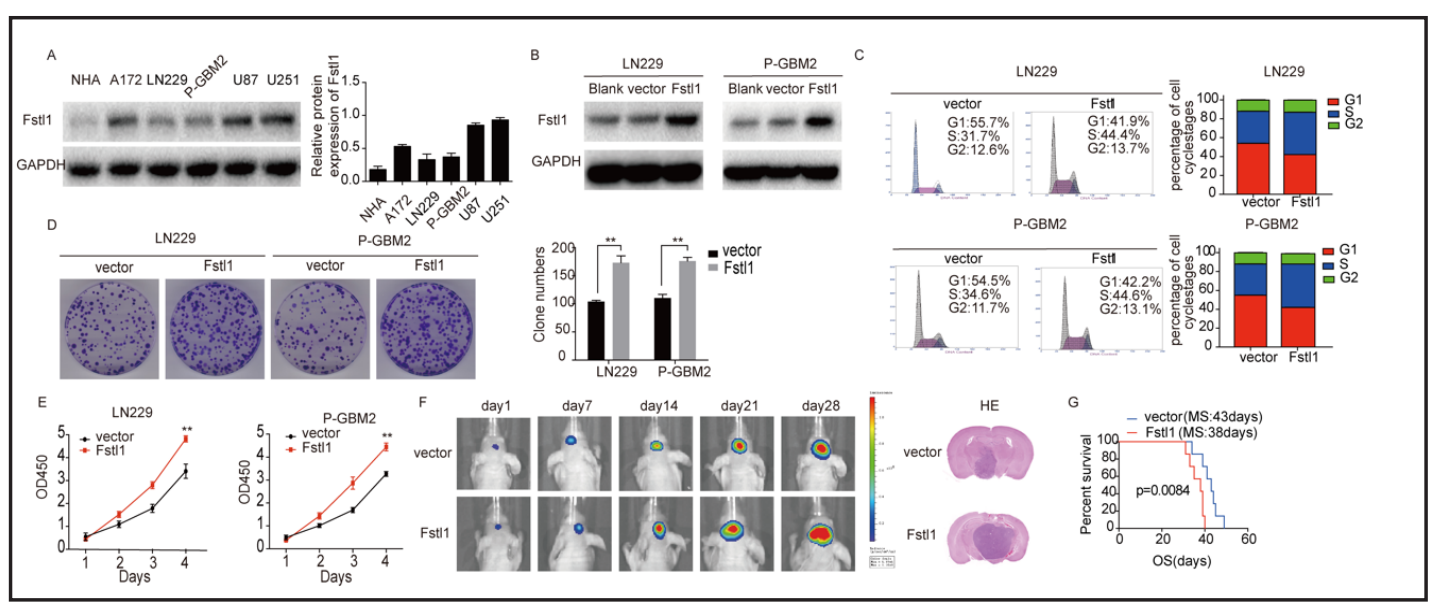

Fig. 2. Fstl1 upregulation promoted glioma growth. (A), Fstl1 expression in various cell lines was assessed by western blotting. (B), Western blot analysis of Fstl1 levels in LN229 and P-GBM2 cells transfected with Fstl1 plasmid or vector control. (C), Cell cycle analyses were performed in LN229 and P-GBM2 cells transfected with Fstl1 plasmid or vector control. (D), Colony formation assays were performed in LN229 and P-GBM2 cells after Fstl1 or vector transduction. (E), Cell proliferation in LN229 and P-GBM2 cells transfected with Fstl1 plasmid or vector control was assessed by CCK8 assays. (F), Representative pseudocolor bioluminescence images of intracranial xenografts of P-GBM2 cells overexpressing Fstl1 or vector obtained on the indicated days. Representative H\&E staining showing tumor cytostructure. (G) Survival curve for mice bearing xenografts of P-GBM2 cells overexpressing Fstl1 or vector. NHAs: normal human astrocytes; P-GBM2: primary GBM2. Data are the means \pm SEM $\left(\mathrm{n}=3,{ }^{* *}, \mathrm{P}<0.01\right)$.

from 54.5 to $42.2 \%$ in the P-GBM2 cell line. The percentage of cells in S phase increased from 31.7 to $44.4 \%$ in the LN229 cell line and from 34.6 to $44.6 \%$ in the P-GBM2 cell line. These findings suggested that Fstl1 accelerated cell transition from the G1/G0 phase to the S phase (Fig. 2C). We also explored the effects of Fstl1 on cell proliferation using a colony formation assay. Fstl1 overexpression increased cell colony formation compared with that in the control group (Fig. 2D). According to CCK-8 assays, Fstl1 overexpressing cells had higher cell proliferation rates than those of the control groups in both LN229 and P-GBM2 cell lines (Fig. 2E). To further examine whether Fstl1 could promote tumor growth in vivo, we intracranially injected P-GBM2 cells expressing Fstl1 or a control vector into immunocompromised mice. Mice injected with P-GBM2 cells expressing Fstl1 had significantly greater tumor volumes than those injected with vector control expressing cells (Fig. 2F). The median survival of the group overexpressing Fstl 1 was $38 \mathrm{~d}$, whereas that of the vector controls was $43 \mathrm{~d}$ (n=5 per group, $P<0.01$; Fig. 2G). These findings indicated that Fstl1 overexpression promoted cell proliferation and cell cycle progression in vitro and in vivo.

\section{Fstl1 knockdown suppressed glioma cell proliferation and cell cycle progression}

The silencing efficacies of Fstl1 shRNA-5/6/7 were compared by western blotting (Fig. 3A). shFstl1-7 had a higher knockdown efficiency than that of the other shRNAs tested in U251 and U87 cells. We used flow cytometry to detect the proportion of transfected cells in the G1/G0 and S phases. Significantly, for shFstl1-7, the proportion of cells in the G1/ G0 phase increased from 54.8 to $73.3 \%$ in the U87 cell line and from 54.5 to $75.1 \%$ in the U251 cell line. The proportion of cells in S phase decreased from 32.9 to $11.2 \%$ in the U87 cell line and from 32.1 to $10.1 \%$ in the U251 cell line. These results indicated that cells transfected with shFstl1-7 were arrested in the G1/G0 phase (Fig. 3B). The colony formation ability of cells transfected with shFstl1-7 was attenuated compared with that in the shCtrl cells (Fig. 3C). The CCK-8 assay showed that the proliferation capacity of cells transfected with shFstl1-7 was less than that of the cells transfected with shCtrl (Fig. 3D). To examine the role of Fstl1 in vivo, we intracranially injected U87 cells expressing shFstl1-7 or shCtrl 


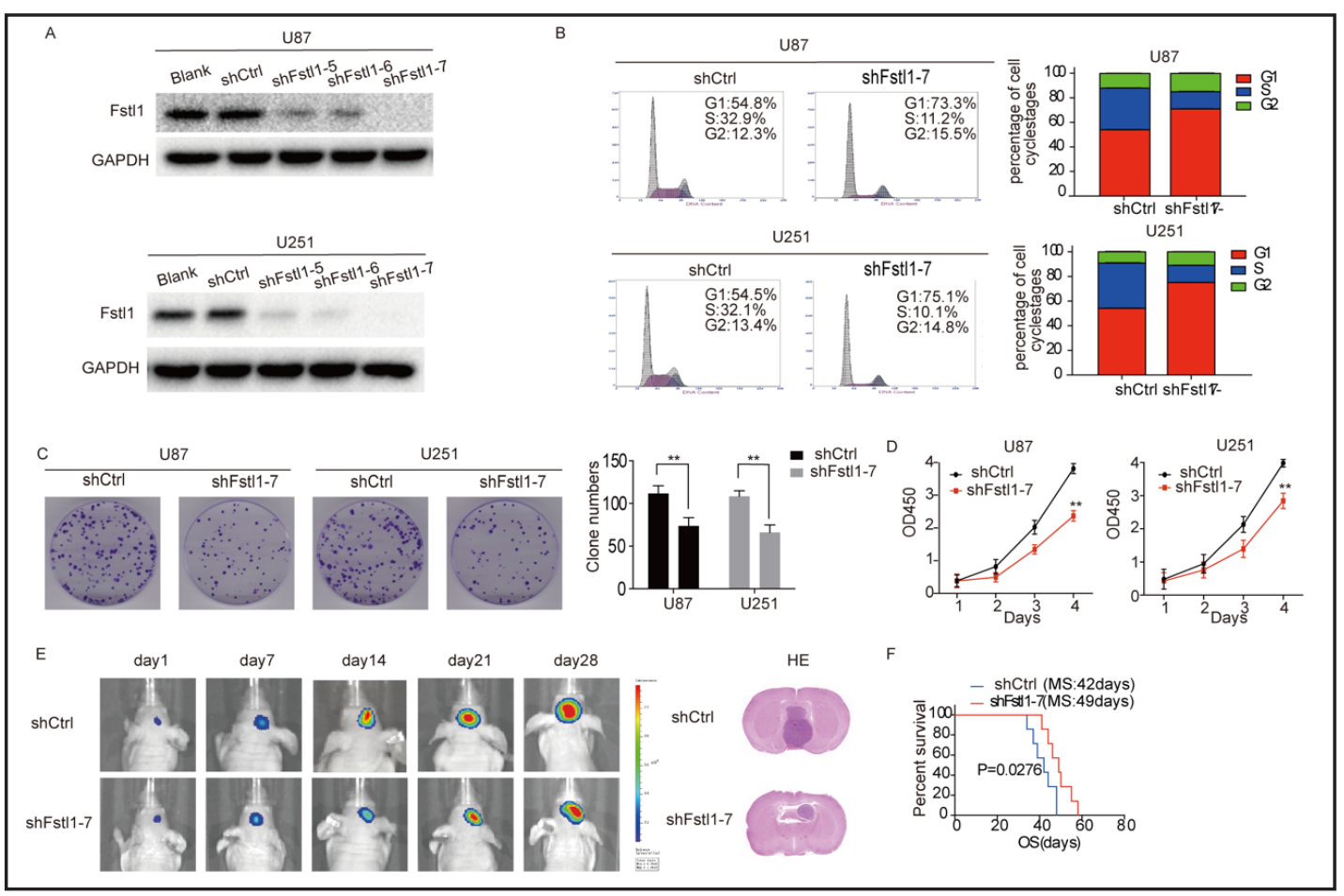

Fig. 3. Fstl1 downregulation attenuated glioma growth. (A), Fstl1 expression in U87 and U251 cells transfected with shRNA-5, shRNA-6 or shRNA-7. (B), Cell cycle analyses were performed in U87 and U251 cells transfected with shFstl1-7, and the results are compared with those in cells transfected with shCtrl. (C), Colony formation assays were performed in U87 and U251 cells after Fstl1 or vector transduction. (D) Proliferation in U87 and U251 cells transfected with shFstl1-7 or shCtrl via CCK8 assays. (E) Representative pseudocolor bioluminescence images of intracranial xenografts of U87 cells on the indicated days. Representative H\&E staining showing tumor cytostructure. (F) Survival curves for mice with intracranial xenografts of U87 cells transfected with shFstl1-7 or shCtrl. Data are the means \pm SEM $(n=3, * *, P<0.01)$.

into immunocompromised mice. Mice injected with U87 cells expressing shFstl1-7 had significantly decreased tumor volumes compared with those in the shCtrl expression group (Fig. 3E). The median survival of mice in the shFstl1-7-treated group was $49 \mathrm{~d}$, whereas that of the shCtrl-treated group was $42 \mathrm{~d}$ ( $\mathrm{n}=5$ per group, $P<0.01$; Fig. $3 \mathrm{~F}$ ). Our findings demonstrated that Fstl1 knockdown attenuated cell proliferation in vitro and in vivo.

\section{Fstl1 impaired the association between BMP4 and BMPR2 by competitively binding with BMP4}

Fstl1 was reported to bind to BMP4, which, in turn, normally binds to BMPR2 in the TGF- $\beta$ signaling pathway [32]. To further evaluate the relationship between BMP4 and BMPR2, we performed co-immunoprecipitation experiments to explore the interactions among Fstl1, BMP4 and BMPR2. Fstl1 and BMPR2 co-immunoprecipitated with BMP4 (Fig. 4A). However, BMPR2 did not co-immunoprecipitate with Fstl1 (Figs. 4B-C). Furthermore, Fstl1 overexpression significantly increased the interaction between Fstl1 and BMP4 and decreased that between BMP4 and BMPR2 (Fig. 4D). Moreover, compared with binding in the control cells, BMP4 binding to BMPR2 was significantly increased when Fstl1 was downregulated, and BMP4 binding to Fstl1 was decreased (Fig. 4E). Therefore, Fstl1 inhibited the association between BMP4 and BMPR2 by competitively binding to BMP4. We next investigated whether Fstl1 could affect the BMP4/Smad1/5/8 signaling pathway. BMP4 is known to bind to heteromultimers of type I and type II BMPRs and to activate the canonical Smad signaling pathway; therefore, we examined the activation-related phosphorylation of 


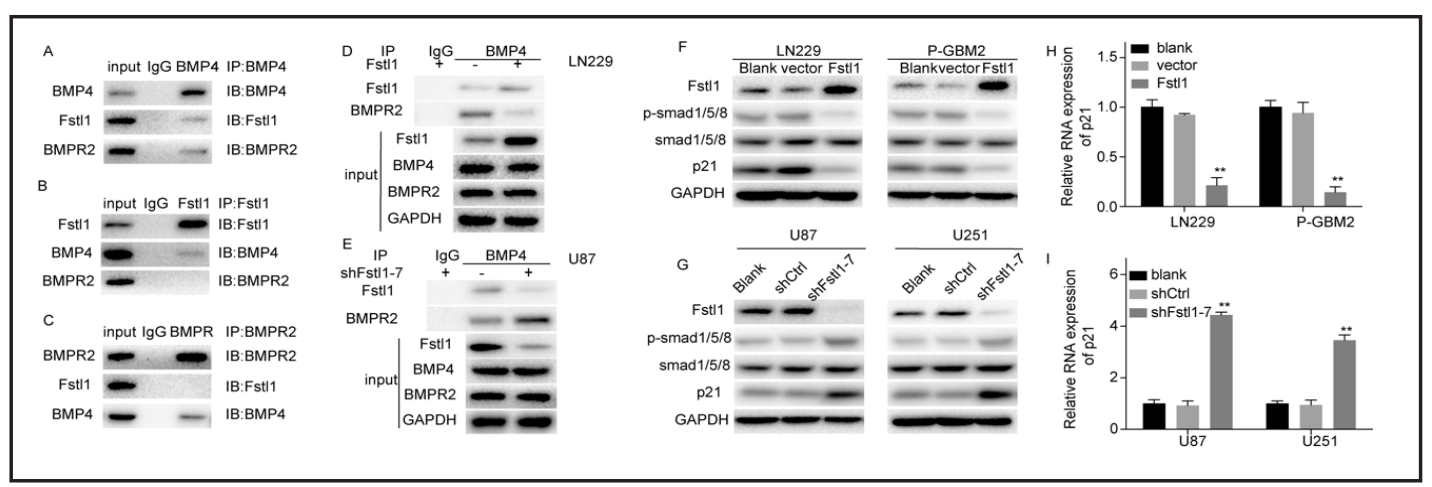

Fig. 4. Fstl1 inhibited Smad1/5/8 phosphorylation by blocking the association between BMP4 and BMPR2 via competitive binding to BMP4. (A-C), Lysates from U87 cells were incubated with anti-IgG, anti-Fstl1, anti-BMP4 or anti-BMPR2 antibody. Immunoprecipitated material was subjected to SDS-PAGE and western blotting with anti-Fstl1, anti-BMP4 and anti-BMPR2 antibodies. (D) and (E), Co-IP was performed using lysates prepared from U87 cells transfected with Fstl1 or vector control and anti-IgG or anti-BMP4 antibody. Western blotting was performed with the indicated antibodies. (F), Levels of phosphorylated Smad1/5/8 (p-Smad1/5/8) and total Smad1/5/8 and p21 protein in cells overexpressing Fstl1 or vector. (G), Levels of phosphorylated Smad1/5/8 (p-Smad1/5/8) and total Smad1/5/8 and p21 protein in cells transfected with shFstl1-7 or shCtrl. (H), Relative RNA expression of p21 in GBM cells overexpressing Fstl1 was detected by qRT-PCR. (I), Relative RNA expression of p21 in cells transfected with shFstl-7 or shCtrl was detected by qRT-PCR. Data are the means \pm SEM $(* * \mathrm{P}<0.01)$.

Smad1/5/8 and the levels of p21, a protein downstream of Smad1/5/8. Immunoblotting showed that Smad1/5/8 phosphorylation and p21 levels were decreased in LN229 and P-GBM2 cells overexpressing Fstl1, while the total Smad1/5/8 levels were not affected (Fig. $4 \mathrm{~F}$ ). In addition, compared with those in the corresponding control cells, phosphorylated Smad1/5/8 (p-Smad1/5/8) and p21 levels were increased in U87 and U251 cells in which Fstl1 was downregulated, but Smad1/5/8 expression levels were not affected (Fig. 4G). According to the qRT-PCR results, p21 expression was decreased in cells overexpressing Fstl1 but was increased in cells with Fstl1 downregulated (Fig. 4H). These results suggested that Fstl1 competitively bound to BMP4, decreasing BMP4-BMPR2 binding and thus regulating the Smad1/5/8 signing pathway.

\section{BMP4 overexpression reversed the inhibitory effects of Fstl1}

To further investigate whether Fstl1 inhibited Smad1/5/8 signaling through competitive binding with BMP4, we overexpressed Fstl1 and BMP4 together in LN229 and P-GBM2 cells and analyzed the levels of $\mathrm{p}-\mathrm{Smad} 1 / 5 / 8, \mathrm{Smad} 1 / 5 / 8$ and $\mathrm{p} 21$ by western blotting. The effects of Fstl1 overexpression on these proteins were reversed by high levels of BMP4 expression but not low levels of BMP4 expression (Figs. 5A-C). To further confirm the mechanism through which Fstl1 regulates cell cycle progression, GBM cells were cotransfected with Fstl1 and BMP4 plasmids. In addition, the ectopic expression of BMP4 significantly abrogated the effects of Fstl1 on cell cycle progression (Fig. 5D). Similarly, the increased proliferation in LN229 and P-GBM2 cells induced by Fstl1 was also restored by BMP4 overexpression (Figs. 5E-F). To determine the clinical relevance of the associations among Fstl1, p-Smad1/5/8 and p21, we performed IHC staining of these three proteins in glioma tissue samples from two GBM patients. There was a significant negative correlation between the levels of Fstl1 and the other two proteins in these GBM specimens. Tumors with low levels of Fstl1 tended to express high levels of $\mathrm{p}$-Smad1/5/8 and p21, whereas those with high Fstl1 levels tended to express low levels of p-Smad1/5/8 and p21 (Fig. 5G). Based on these findings, we concluded that Fstl1 could regulate glioma growth by competitively binding to BMP4 and inhibiting the Smad1/5/8 signaling pathway. 


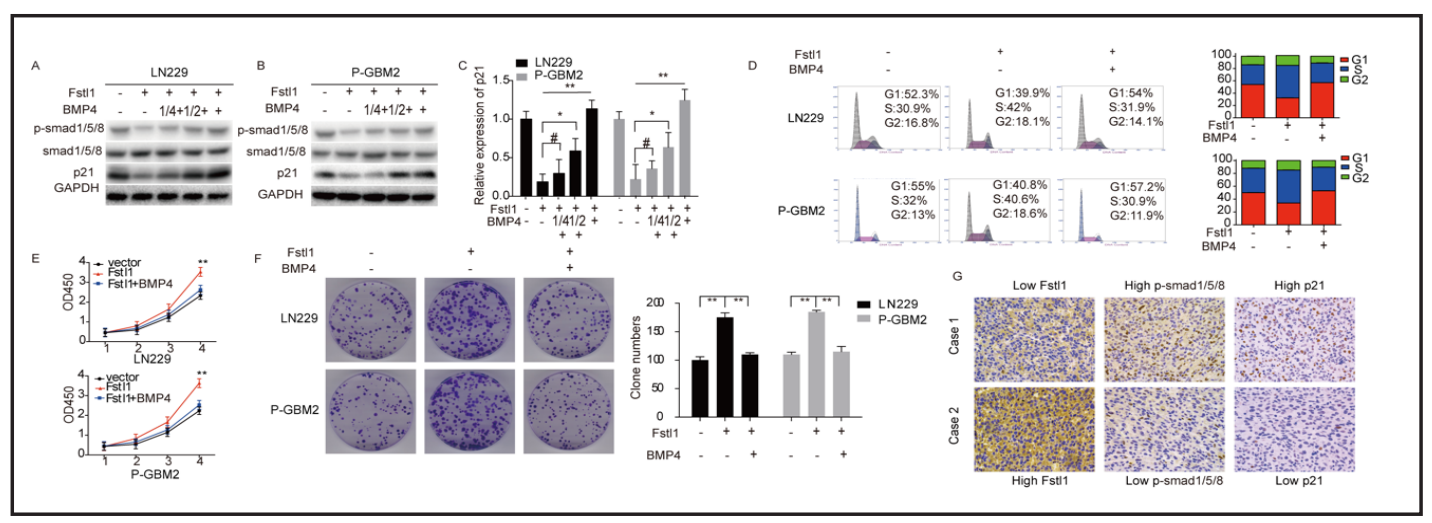

Fig. 5. BMP4 reintroduction reversed the inhibitory effects of Fstl1. (A) and (B), Levels of p-Smad1/5/8, Smad1/5/8 and p21 in cells changed in accordance with gradual changes in BMP4 levels as detected by western blot analyses. (C), Relative RNA expression levels of p21 in LN229 and U87 cells detected by qRTPCR. Data are the means \pm SEM $(* * P<0.01, \# P>0.05)$. (D), Cell cycle analyses showed that the percentage of cells in G1/G0 phase was normalized after BMP4 reintroduction. (E), Proliferation of LN229 and U87 cells assessed by CCK8 assays was significantly changed in response to changes in BMP4 expression. (F), Colony formation assays performed in Ln229 and U87 cells after BMP4 reintroduction. (G) IHC staining of Fstl1, p-Smad1/5/8 and p21 in two representative GBM specimens from the tissue microarrays. Brown staining indicates positive immunoreactivity. Scale bar, $50 \mathrm{~mm}$. Data are the means $\pm \operatorname{SEM}(\mathrm{n}=3, * *, \mathrm{P}<0.01 ; \#, \mathrm{P}>$ 0.05).

\section{Discussion}

GBMs are the most malignant form of astrocytomas. Several genetic alterations in GBMs have been described, but the prognosis of glioma patients remains poor due to the fast proliferation rate of glioma cells, short course of the disease, difficulty of early diagnosis, unique position of the lesions, incomplete resection and low sensitivity to chemotherapy and radiotherapy [33-36].

In this study, we used in vitro and in vivo experiments, as well as clinical sample analyses, to show that Fstl1 is a novel key factor responsible for glioma growth. Importantly, Fstl1 expression was significantly correlated with tumor progression and associated with the functional performance of glioma cells. Our experiments showed that the overexpression of Fstl1 promoted G1/G0 to $S$ phase transformation in glioma cells. Glioma cell proliferation was upregulated as well. At the same time, Kaplan-Meier curves showed that the mean survival of nude mice was significantly decreased in the Fstl1 overexpression group in comparison with that of the vector control-treated group. Likewise, targeted inactivation of the Fstl1 gene suppressed cell cycle progression and glioma cell proliferation, ultimately decreasing tumor-related functions. In other words, Fstl1 plays an important role in glioma growth.

However, the mechanism by which Fstl1 regulates cell cycle progression and proliferation remains unclear. Fstl1, first identified as a TGF- $\beta 1$ inducible gene, encodes a secreted extracellular glycoprotein that contains a follistatin-like domain in its amino acid sequences [23-25]. Fstl1 has a domain similar to follistatin. In addition, its orthologue in zebrafish, zfstl 1/2, functions analogously to nog1 and chd to antagonize BMP activity during zebrafish development [37-39]. Previous reports show that Fstl1 can bind to BMP4 with a $K_{d}$ similar to that of follistatin binding to activin [27, 32, 40, 41]. However, the role of Fstl1 in BMP signaling remains unknown in glioma.

Further studies indicated that Fstl1 could combine with BMP4. BMP4, a member of the BMP family, is inherently involved in brain and spinal cord development and is a significant factor in nervous system development [42] that induces neural stem cells to differentiate into either neurons or astrocytes; BMP4 may affect neural stem cell differentiation through

\section{KARGER}


multiple mechanisms [43]. BMP4 was triggered by a heteromeric serine/threonine kinase receptor composed of two type I signal-transducing receptors (BMP Ia and BMPR Ib) and one type II (BMPR II) receptor that bind to RII and then interact with RIa or RIb. Smad transcription factors are important intracellular effectors of the BMP signaling pathway. There are three subclasses based on biological function: receptor-regulated Smads (R-Smad), common-mediator Smads (Co-Smad), and inhibitory Smads (I-Smad)[44]. Activating BMPR specifically mediates the phosphorylation of BMPR-Smads (Smad1, Smad5 and Smad8), which then interact with co-Smad4 to form heterotrimeric complexes that mediate intranuclear signaling to regulate gene expression [45]. However, there is no relationship between Fstl1 and BMPR2. In the current study, Co-IP revealed that BMP4 could combine with Fstl1 and BMPR2, while Fstl1 could not bind to BMPR2. The ectopic expression of Fstl1 impaired the association between BMP4 and BMPR2 because Fstl1 competitively bound to BMP4 to prevent the phosphorylation of the Smad1/5/8 pathways; then, Smad4 was combined in the cytoplasm and translocated into the nucleus, impacting the expression of its downstream genes, e.g., p21. P21, which is also known as cyclin-dependent kinase inhibitor 1 or CDKinteracting protein 1 , is a cyclin-dependent kinase inhibitor that inhibits the complexes of CDK2, CDK1 and CDK4/ 6 and thus functions as a regulator of cell cycle progression at the G1/ $\mathrm{G} 0$ and $\mathrm{S}$ phases [46]. Moreover, the knockdown of Fstl1 expression enhanced the association between BMP4 and BMPR2 and then promoted phosphorylation of the Smad1/5/8 pathways and further increased the expression levels of p21, blocking cell cycle progression at the G1/G0 phase and inhibiting cell proliferation. Otherwise, the overexpression of BMP4 could reverse the effects of Fstl1 in glioma cells. Accordingly, we conclude that Fstl1 can mediate the BMP4/Smad1/5/8 pathway by binding BMP4 competitively.

Overall, our study showed that Fstl1 is a protein critical for modulating cell growth in GBMs. Fstl1 competitively binds to BMP4 to inhibit phosphorylation of the Smad1/5/8 pathways and the expression levels of p21, thus accelerating cell cycle progression and glioma cell proliferation, ultimately facilitating tumor-related functions. Our findings identified a novel mechanism of Fstl1-induced signaling inhibition in GBMs. In this work, we investigated only whether Fstl1 could affect proliferation and cell cycle progression in glioma via the BMP4/Smad1/5/8 signaling pathway. However, there may be many other Fstl1-related signaling pathways that can greatly influence cancer progression, especially in glioma. Further information regarding these Fstl1-induced signaling pathways in glioma progression is needed. In addition, this work has important implications for the development of treatment strategies for glioblastoma.

\section{Abbreviations}

Fstl1 (follistatin-like (1; GBM: glioblastoma multiforme); NBT (nontumor brain tissue); NHAs (normal human astrocytes); GBM (glioblastoma multiforme); BMPs (bone morphogenetic proteins); TGF- $\beta$ (transforming growth factor beta); Smad1/5/8 (small mothers against decapentaplegic 1/5/8); A (astrocytoma); AA anaplastic astrocytoma); AO (anaplastic oligodendroglioma); Mixed (mixed glioma).

\section{Acknowledgements}

We thank Department of Neurosurgery, The First Affiliated Hospital of Nanjing Medical University (Nanjing, China) for its generous help.

This work is supported by the National High Technology Research and Development Program of China (863) (No.2012AA02A508), National Natural Science Foundation of China (81472362, 81072078 and 81272792), The National Key Research and Development Plan (No.2016YFC0902500), grants from Jiangsu Province's Key Provincial Talents Program (No. RC2011051). 


\section{Cellular Physiology Cell Physiol Biochem 2017;44:1616-1628 \begin{tabular}{ll|l} 
and Biochemistry & $\begin{array}{l}\text { DOI: 10.1159/000485759 } \\
\text { Published onlIne: December 06, } 2017\end{array}$ & $\begin{array}{l}\text { (c) } 2017 \text { The Author(s). Published by S. Karger AG, Basel } \\
\text { www.karger.com/cpb }\end{array}$ \\
\hline
\end{tabular}

All data generated or analysed during this study are included in this published article.

Conception and design: X.J. and E.N. Development of methodology: E.N., T.Y and X.J. Acquisition of the data (provided animals, acquired and managed patients, provided facilities and so on): E.N., X.J., A.Z., X.Z., T.Z. Analysis and interpretation of the data: E.N., J.Z. and Y.Y. Writing, review and/or revision of the manuscript: X.J. and E.N. Administrative, technical or material support: E.N. and K.J. Study supervision: Y.Y., Y.W. and X.Z

\section{Disclosure Statement}

The authors declare no competing financial interests. Informed consent was obtained from all individual participants included in the study. All procedures performed in studies involving human participants were in accordance with the ethical standards of the institutional research committee and with the 1964 Helsinki Declaration and its later amendments or comparable ethical standards.

\section{References}

1 Liu N, Zhang L, Wang Z, Cheng Y, Zhang P, Wang X, Wen W, Yang H, Liu H, Jin W, Zhang Y, Tu Y: MicroRNA-101 inhibits proliferation, migration and invasion of human glioblastoma by targeting SOX9. Oncotarget 2016;10.

2 Zhang C, Liu S, Yuan X, Hu Z, Li H, Wu M, Yuan J, Zhao Z, Su J, Wang X, Liao Y, Liu Q: Valproic Acid Promotes Human Glioma U87 Cells Apoptosis and Inhibits Glycogen Synthase Kinase-3beta Through ERK/Akt Signaling. Cell Physiol Biochem 2016;39:2173-2185.

-3 Cao L, Qu D, Wang H, Zhang S, Jia C, Shi Z, Wang Z, Zhang J, Ma J: Toosendanin Exerts an Anti-Cancer Effect in Glioblastoma by Inducing Estrogen Receptor beta- and p53-Mediated Apoptosis. Int J Mol Sci 2016;17:

-4 Huang W, Wang J, Zhang D, Chen W, Hou L, Wu X, Lu Y: Inhibition of KIF14 Suppresses Tumor Cell Growth and Promotes Apoptosis in Human Glioblastoma. Cell Physiol Biochem 2015;37:1659-1670.

-5 Xu RX, Liu RY, Wu CM, Zhao YS, Li Y, Yao YQ Xu YH: DNA damage-induced NF-kappaB activation in human glioblastoma cells promotes miR-181b expression and cell proliferation. Cell Physiol Biochem 2015;35:913-925.

-6 Lai D, Yang X: BMP4 is a novel transcriptional target and mediator of mammary cell migration downstream of the Hippo pathway component TAZ. Cell Signal 2013;25:1720-1728.

7 Yu Y, Ran Q: Nuclear SMAD2 restrains proliferation of glioblastoma. Cell Physiol Biochem 2015;35:17561763.

8 Alarmo EL, Kallioniemi A: Bone morphogenetic proteins in breast cancer: dual role in tumourigenesis? Endocr Relat Cancer 2010;17:R123-139.

-9 Singh A, Morris RJ: The Yin and Yang of bone morphogenetic proteins in cancer. Cytokine Growth Factor Rev 2010;21:299-313.

10 Thawani JP, Wang AC, Than KD, Lin CY, La Marca F, Park P: Bone morphogenetic proteins and cancer: review of the literature. Neurosurgery 2010;66:233-246.

-11 Ivanova T, Zouridis H, Wu Y, Cheng LL, Tan IB, Gopalakrishnan V, Ooi CH, Lee J, Qin L, Wu J, Lee M, Rha SY, Huang D, Liem N, Yeoh KG, Yong WP, Teh BT, Tan P: Integrated epigenomics identifies BMP4 as a modulator of cisplatin sensitivity in gastric cancer. Gut 2013;62:22-33.

$>12$ Kallioniemi A: Bone morphogenetic protein 4-a fascinating regulator of cancer cell behavior. Cancer Genet 2012;205:267-277.

13 Kim SG, Park HR, Min SK, Choi JY, Koh SH, Kim JW, Lee HW: Expression of bone morphogenic protein-4 is inversely related to prevalence of lymph node metastasis in gastric adenocarcinoma. Surg Today 2011;41:688-692.

14 Giacomini D, Paez-Pereda M, Theodoropoulou M, Labeur M, Refojo D, Gerez J, Chervin A, Berner S, Losa M, Buchfelder M, Renner U, Stalla GK, Arzt E: Bone morphogenetic protein-4 inhibits corticotroph tumor cells: involvement in the retinoic acid inhibitory action. Endocrinology 2006;147:247-256. 


\section{Cellular Physiology Cell Physiol Biochem 2017;44:1616-1628 \begin{tabular}{l|l|l} 
DOI: 10.1159/000485759 & $\begin{array}{l}\text { O 2017 The Author(s). Published by S. Karger AG, Basel } \\
\text { www.karger.com/cpb }\end{array}$
\end{tabular} \\ Jin et al.: Fstl1-BMP4-Smad1/5/8 Signaling}

15 Chiu CY, Kuo KK, Kuo TL, Lee KT, Cheng KH: The activation of MEK/ERK signaling pathway by bone morphogenetic protein 4 to increase hepatocellular carcinoma cell proliferation and migration. Mol Cancer Res 2012;10:415-427.

-16 Deng H, Makizumi R, Ravikumar TS, Dong H, Yang W, Yang WL: Bone morphogenetic protein-4 is overexpressed in colonic adenocarcinomas and promotes migration and invasion of HCT116 cells. Exp Cell Res 2007;313:1033-1044.

-17 Johnson MD, O'Connell MJ, Vito F, Pilcher W: Bone morphogenetic protein 4 and its receptors are expressed in the leptomeninges and meningiomas and signal via the Smad pathway. J Neuropathol Exp Neurol 2009;68:1177-1183.

18 Guo D, Huang J, Gong J: Bone morphogenetic protein 4 (BMP4) is required for migration and invasion of breast cancer. Mol Cell Biochem 2012;363:179-190.

19 Hjertner 0, Hjorth-Hansen H, Borset M, Seidel C, Waage A, Sundan A: Bone morphogenetic protein-4 inhibits proliferation and induces apoptosis of multiple myeloma cells. Blood 2001;97:516-522.

20 Park ES, Woods DC, Tilly JL: Bone morphogenetic protein 4 promotes mammalian oogonial stem cell differentiation via Smad1/5/8 signaling. Fertil Steril 2013;100:1468-1475.

21 Feng J, Gao J, Li Y, Yang Y, Dang L, Ye Y, Deng J, Li A: BMP4 enhances foam cell formation by BMPR-2/ Smad1/5/8 signaling. Int J Mol Sci 2014;15:5536-5552.

22 Wu Q, Yao J: BMP4, a new prognostic factor for glioma. World J Surg Oncol 2013;11:264.

23 Chaly Y, Hostager B, Smith S, Hirsch R: Follistatin-like protein 1 and its role in inflammation and inflammatory diseases. Immunol Res 2014;59:266-272.

24 Bae K, Park KE, Han J, Kim J, Kim K, Yoon KA: Mitotic cell death caused by follistatin-like 1 inhibition is associated with up-regulated Bim by inactivated Erk1/2 in human lung cancer cells. Oncotarget 2016;7:18076-18084.

25 Liu R, Yang Y, Shen J, Chen H, Zhang Q, Ba R, Wei Y, Li KC, Zhang X, Zhao C: Fstl1 is involved in the regulation of radial glial scaffold development. Mol Brain 2015;8:53.

-26 Gold E, Risbridger G: Activins and activin antagonists in the prostate and prostate cancer. Mol Cell Endocrinol 2012;359:107-112.

27 Wu J, Dong Y, Teng X, Cheng M, Shen Z, Chen W: Follistatin-like 1 attenuates differentiation and survival of erythroid cells through Smad2/3 signaling. Biochem Biophys Res Commun 2015;466:711-716.

28 Chaly Y, Blair HC, Smith SM, Bushnell DS, Marinov AD, Campfield BT, Hirsch R: Follistatin-like protein 1 regulates chondrocyte proliferation and chondrogenic differentiation of mesenchymal stem cells. Ann Rheum Dis 2015;74:1467-1473.

29 Serao NV, Delfino KR, Southey BR, Beever JE, Rodriguez-Zas SL: Cell cycle and aging, morphogenesis, and response to stimuli genes are individualized biomarkers of glioblastoma progression and survival. BMC Med Genomics 2011;4:49.

-30 Reddy SP, Britto R, Vinnakota K, Aparna H, Sreepathi HK, Thota B, Kumari A, Shilpa BM, Vrinda M, Umesh S, Samuel C, Shetty M, Tandon A, Pandey P, Hegde S, Hegde AS, Balasubramaniam A, Chandramouli BA, Santosh V, Kondaiah P, Somasundaram K, Rao MR: Novel glioblastoma markers with diagnostic and prognostic value identified through transcriptome analysis. Clin Cancer Res 2008;14:2978-2987.

-31 Nie E, Jin X, Wu W, Yu T, Zhou X, Zhi T, Shi Z, Zhang J, Liu N, You Y: BACH1 Promotes Temozolomide Resistance in Glioblastoma through Antagonizing the Function of p53. Sci Rep 2016;6:39743.

32 Geng Y, Dong Y, Yu M, Zhang L, Yan X, Sun J, Qiao L, Geng H, Nakajima M, Furuichi T, Ikegawa S, Gao X, Chen YG, Jiang D, Ning W: Follistatin-like 1 (Fstl1) is a bone morphogenetic protein (BMP) 4 signaling antagonist in controlling mouse lung development. Proc Natl Acad Sci U S A 2011;108:7058-7063.

33 Hutterer M, Gunsilius E, Stockhammer G: Molecular therapies for malignant glioma. Wien Med Wochenschr 2006;156:351-363.

34 Wang H, Zhang Y, Bai R, Wang M, Du S: Baicalin Attenuates Alcoholic Liver Injury through Modulation of Hepatic Oxidative Stress, Inflammation and Sonic Hedgehog Pathway in Rats. Cell Physiol Biochem 2016;39:1129-1140.

-35 Tseliou M, Al-Qahtani A, Alarifi S, Alkahtani SH, Stournaras C, Sourvinos G: The Role of RhoA, RhoB and RhoC GTPases in Cell Morphology, Proliferation and Migration in Human Cytomegalovirus (HCMV) Infected Glioblastoma Cells. Cell Physiol Biochem 2016;38:94-109. 


\section{Cellular Physiology Cell Physiol Biochem 2017;44:1616-1628 \begin{tabular}{l|l|l} 
DOI: 10.1159/000485759 & $\begin{array}{l}\text { O 2017 The Author(s). Published by S. Karger AG, Basel } \\
\text { www.karger.com/cpb }\end{array}$
\end{tabular} \\ Jin et al.: Fstl1-BMP4-Smad1/5/8 Signaling}

-36 Li C, Liu Y, Liu H, Zhang W, Shen C, Cho K, Chen X, Peng F, Bi Y, Hou X, Yang Z, Zheng Z, Wang K, Wang X, Zhang J, Zhong C, Zou H, Zhang X, Zhao S: Impact of autophagy inhibition at different stages on cytotoxic effect of autophagy inducer in glioblastoma cells. Cell Physiol Biochem 2015;35:1303-1316.

-37 Lovely CB, Swartz ME, McCarthy N, Norrie JL, Eberhart JK: Bmp signaling mediates endoderm pouch morphogenesis by regulating Fgf signaling in zebrafish. Development 2016;143:2000-2011.

-38 Sarmah S, Muralidharan P, Marrs JA: Embryonic Ethanol Exposure Dysregulates BMP and Notch Signaling, Leading to Persistent Atrio-Ventricular Valve Defects in Zebrafish. PLoS One 2016;11:e0161205.

-39 Khatri D, Zizioli D, Tiso N, Facchinello N, Vezzoli S, Gianoncelli A, Memo M, Monti E, Borsani G, Finazzi D: Down-regulation of coasy, the gene associated with NBIA-VI, reduces Bmp signaling, perturbs dorso-ventral patterning and alters neuronal development in zebrafish. Sci Rep 2016;6:37660.

40 Chen W, Xia J, Hu P, Zhou F, Chen Y, Wu J, Lei W, Shen Z: Follistatin-like 1 protects cardiomyoblasts from injury induced by sodium nitroprusside through modulating Akt and Smad1/5/9 signaling. Biochem Biophys Res Commun 2016;469:418-423.

41 Javerzat S, Franco M, Herbert J, Platonova N, Peille AL, Pantesco V, De Vos J, Assou S, Bicknell R, Bikfalvi A, Hagedorn M: Correlating global gene regulation to angiogenesis in the developing chick extra-embryonic vascular system. PLoS One 2009;4:e7856.

42 Watanabe M, Kang YJ, Davies LM, Meghpara S, Lau K, Chung CY, Kathiriya J, Hadjantonakis AK, Monuki ES: BMP4 sufficiency to induce choroid plexus epithelial fate from embryonic stem cell-derived neuroepithelial progenitors. J Neurosci 2012;32:15934-15945.

43 Zhou Z, Chen ZW, Yang XH, Shen L, Ai XH, Lu S, Luo QQ: Establishment of a biomarker model for predicting bone metastasis in resected stage III non-small cell lung cancer. J Exp Clin Cancer Res 2012;31:34.

44 Heldin CH, Miyazono K, ten Dijke P: TGF-beta signalling from cell membrane to nucleus through SMAD proteins. Nature 1997;390:465-471.

45 Fang H, Song P, Shen C, Liu X, Li H: Bone mesenchymal stem cell-conditioned medium induces the upregulation of Smad6, which inhibits the BMP-4/Smad1/5/8 signaling pathway. Neurol Res 2016;38:965972.

46 Gartel AL, Radhakrishnan SK: Lost in transcription: p21 repression, mechanisms, and consequences. Cancer Res 2005;65:3980-3985. 